\section{TEM Sample Preparation Using Focused Ion Beam - Capabilities And Limits}

H.J. Engelmann, B. Volkmann, Y. Ritz, H. Saage,

H. Stegmann, Q. de Robillard, E. Zschech

AMD Saxony LLC \& Co. KG, Dresden, Germany

hans-juergen.engelmann@amd.com

TEM sample preparation using Focused Ion Beam (FIB) methods becomes more and more interesting for microscopists because the technique allows for reliable and very efficient sample preparation. The first application of TEM sample preparation by FIB-cutting was reported more than 10 years ago [1]. Meanwhile, a lot of experience has been gathered that allows one to discuss the capabilities and limits of the FIB technique in detail.

Several TEM sample preparation techniques are known that all include FIB-cutting but differ in sample pre-preparation, sample handling, etc. [2]. This paper focuses on the actual FIB process. FIB tools are closely related to Scanning Electron Microscopes, but instead of an electron beam an ion beam (mostly $\mathrm{Ga}^{+}$ions) is used to remove and deposit material. Additionally, ion-induced secondary electrons can be used for imaging. A detailed description of the design and the function- was completed within $65 \mathrm{~min}$ (30 min mechanical pre-preparation, 35 min FIB-cutting) whereas the classical cross-section preparation took about 4.5 hours.

Another important advantage of FlB-cutting is that target (selected area) preparation is made easier by ion-induced secondary electron (SE) imaging. Thus, the region of interest can be found very easily. But, due to the ion beam exposure, ion-induced SE imaging is always connected with surface modifications like material removal, ion implantation, amorphization etc. so that the interesting structure can be modified or destroyed. These effects can be minimized by carefully utilizing correct procedures.

FIB-cutting usually starts with the deposition of a metal bar that serves as a cutting edge by ion beam assisted deposition from gaseous $W$ or Pt compounds. There is a high impact of the ion beam on to the material while depositing the cutting edge. Modification or damage of the near-surface region can be avoided or at least mitigated by depositing a thin protective layer of about $50 \mathrm{~nm}$, e.g. silicon oxide, nitride or carbon. In the case of the two-beam FIB (combination of electron and ion beam), the electron beam can be used to deposit the PtW cutting edge. Planar surfaces do not need further pre-preparation. For rough surfaces or open structures (e.g. holes, trenches), a 'refill technique' has been developed that uses epoxy resin, that can be cut by the ion beam [5]. An application of the 'refill-technique' is shown in
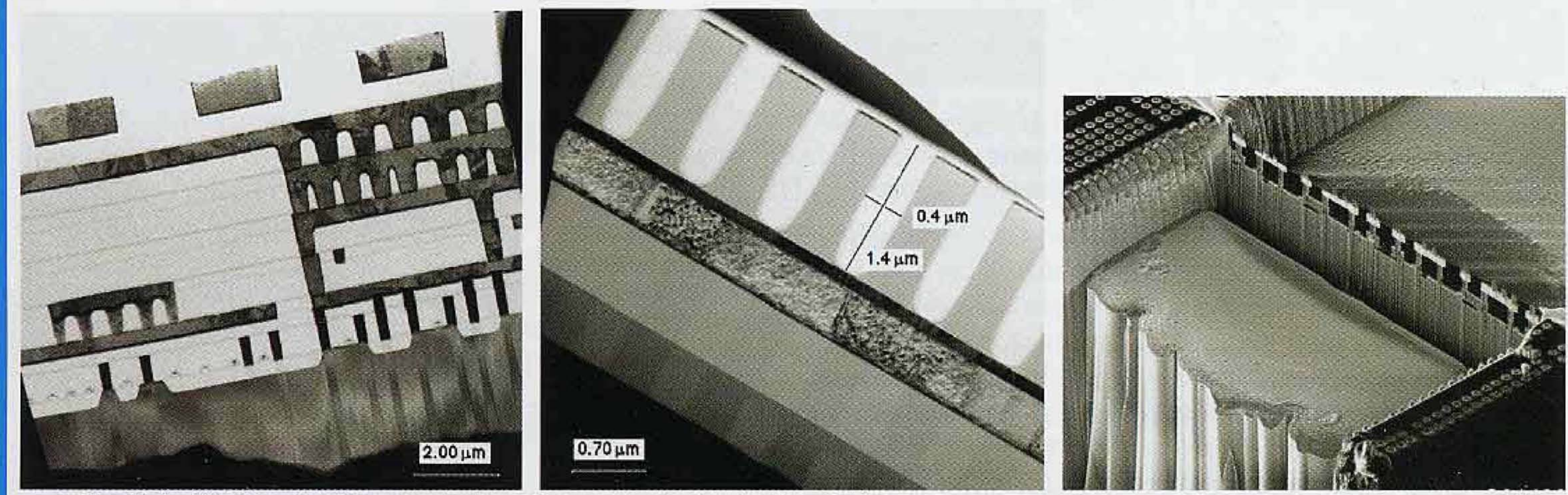

Fig. 1: (Left) FIB-cut TEM cross-section of a semiconductor device structure.

Fig. 2: (Center) FIB-cut TEM cross-section of a trench structure. The trenches were filled by epoxy using the 'refill technique'.

Fig. 3: (Right) FIB-cut TEM cross-section of a semiconductor device structure. The 'curtaining effect' caused by tungsten contacts is clearly visible.

ality of FIB tools is given in [3]. All results shown here were obtained using the conventional "H-bar" FIB technique (FIB-cut in a sample bar mounted on a TEM grid) applying a FEI FIB200TEM tool.

It has been demonstrated that FIB-cutting can be applied to a large variety of materials, among them metals, insulators, organic materials and geological samples. One of the most important advantages of FIB-cutting is that relatively large-area electron transparent TEM lamellae can be prepared even from structures consisting of different materials. As an example, Fig. 1 shows a TEM cross-section of a semiconductor device structure containing various materials.

FIB-cutting is widely used for TEM sample preparation, especially in the semiconductor industry, because this technique provides for short preparation times, and a success rate close to $100 \%$. It has been shown that TEM target preparation can be done within one hour [4]. In this study, a conventional FIB preparation (consisting of mechanical pre-preparation and FIB-cutting) of a structure typically for semiconductor industry is compared with a classical TEM crosssection preparation (consisting of cutting, grinding, dimpling and Ar+ ion-milling) of the same structure. The conventional FIB preparation
Fig. 2. Using the epoxy, a planar surface could be obtained that was necessary for the subsequent cutting edge deposition.

Material removal is done using ion beams with different probe currents. High probe currents are used for rough sputtering since they have a high removal rate. Because of their relatively broad beam profile, low probe currents must be applied for final polishing of the TEM lamella. Since some materials are removed faster than others, the so called 'curtaining effect' is observed that represents regions that remain thicker below structures with low sputter coefficient. The curtaining effect caused by tungsten material can be seen in Fig. 3 .

A high coplanarity of the TEM lamellae can be achieved. Depending on the height of the TEM lamella, the FIB sample has to be tilted during the final cutting steps up to 1 degree. Fig. 4 shows a cross-section of a nearly plan-parallel FIB-cut TEM lamella. Such cross-sections can be obtained using the 'refill technique' mentioned above.

In principle, there is no limit for the final thickness of the TEM lamella. Using low probe currents and alternating polishing from both sides, lamellae of only some $\mathrm{nm}$ thickness can be prepared. However, local mechanical stress states tend to bend thin samples during the 


\section{Imaging beyond imagination - \\ LEO Extended Pressure SEM}

\section{LEO 1400EP}

The natural choice for wet and delicate specimens.
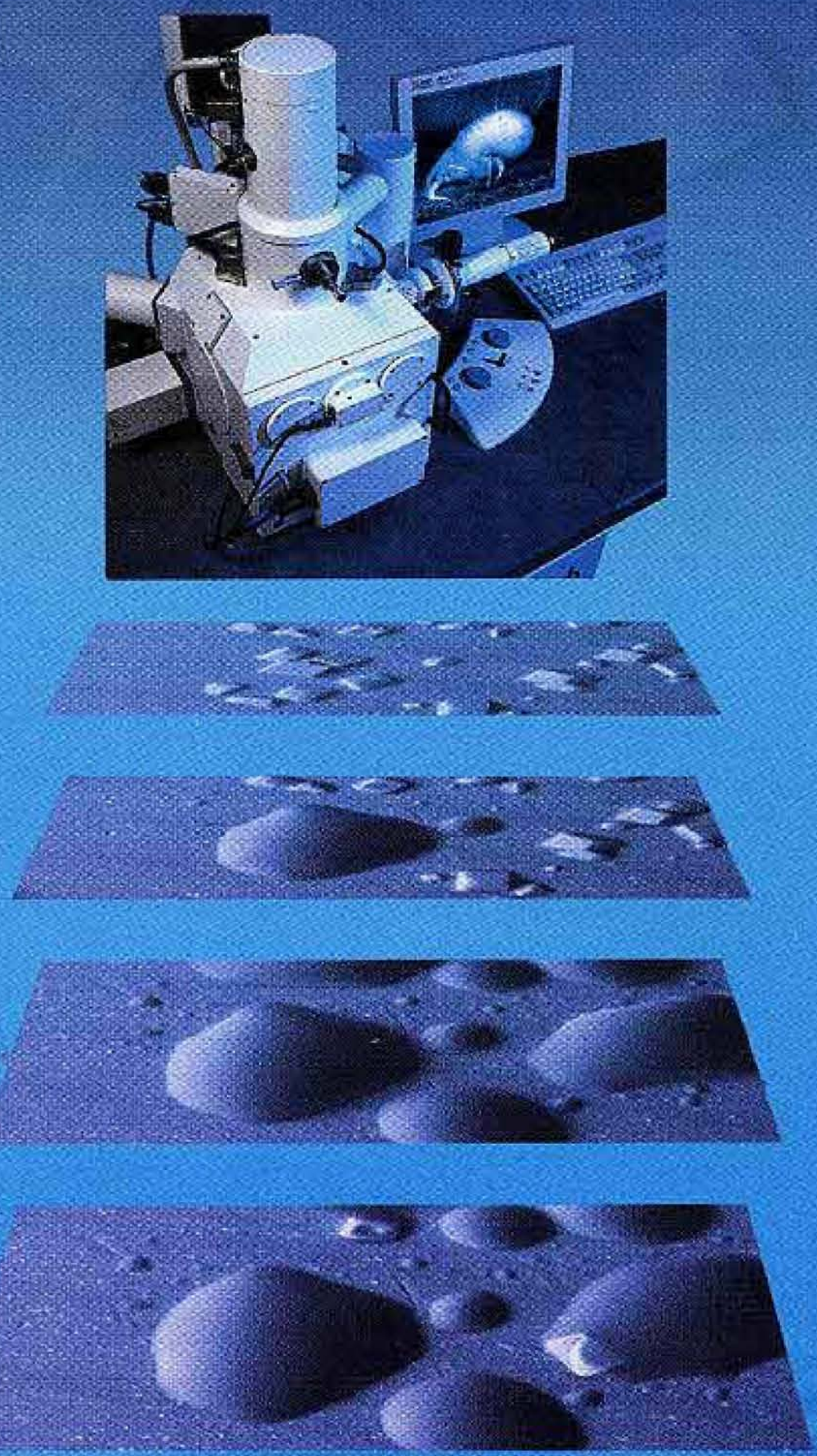

The new LEO 1400EP series of extended pressure scanning electron microscopes enable you to dynamically image wet and delicate samples in real time. It gives you accurate control of chamber water vapor pressure and specimen temperature. This permits one-of-a-kind imaging in life science, pharmaceutical and material science applications.

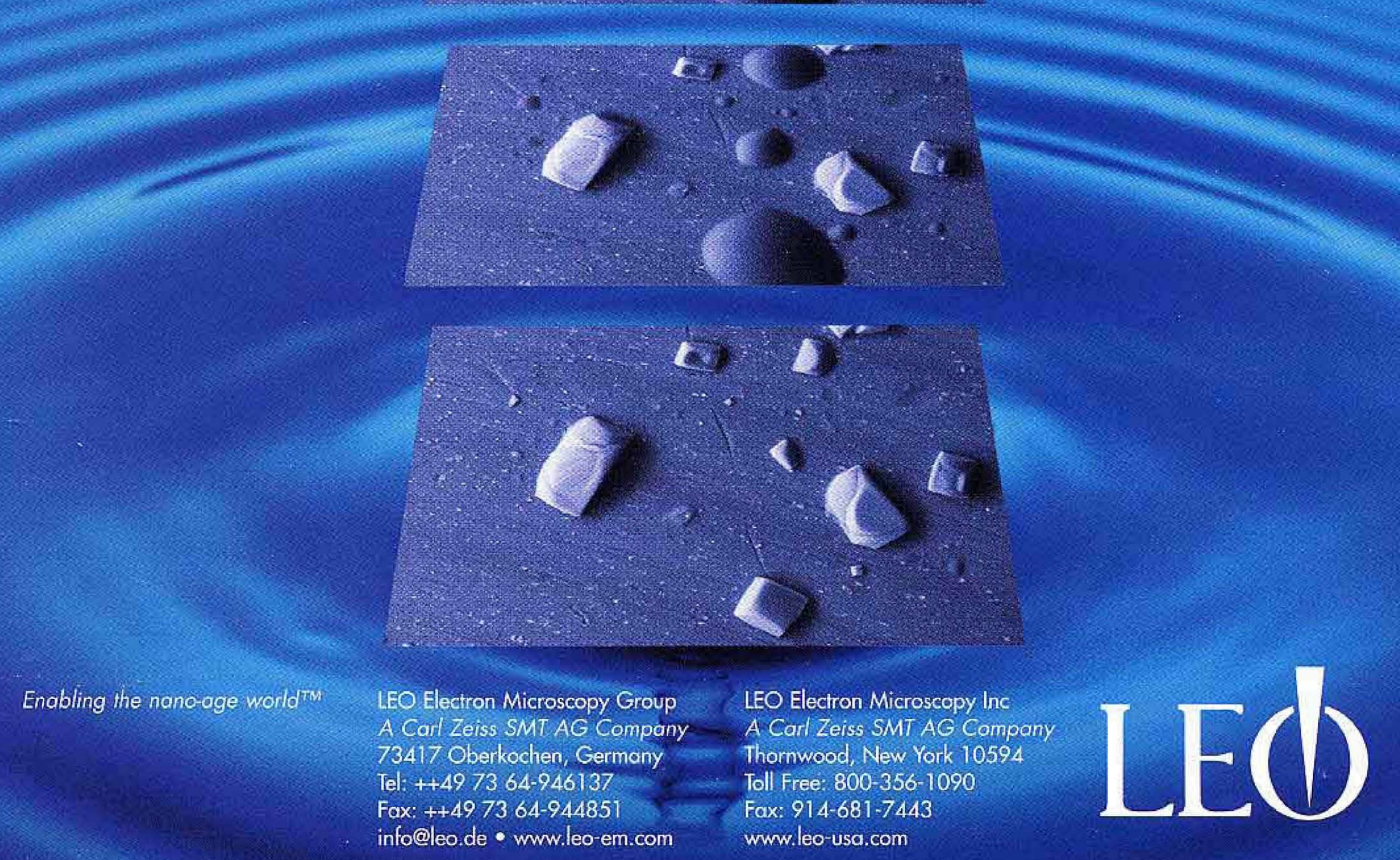




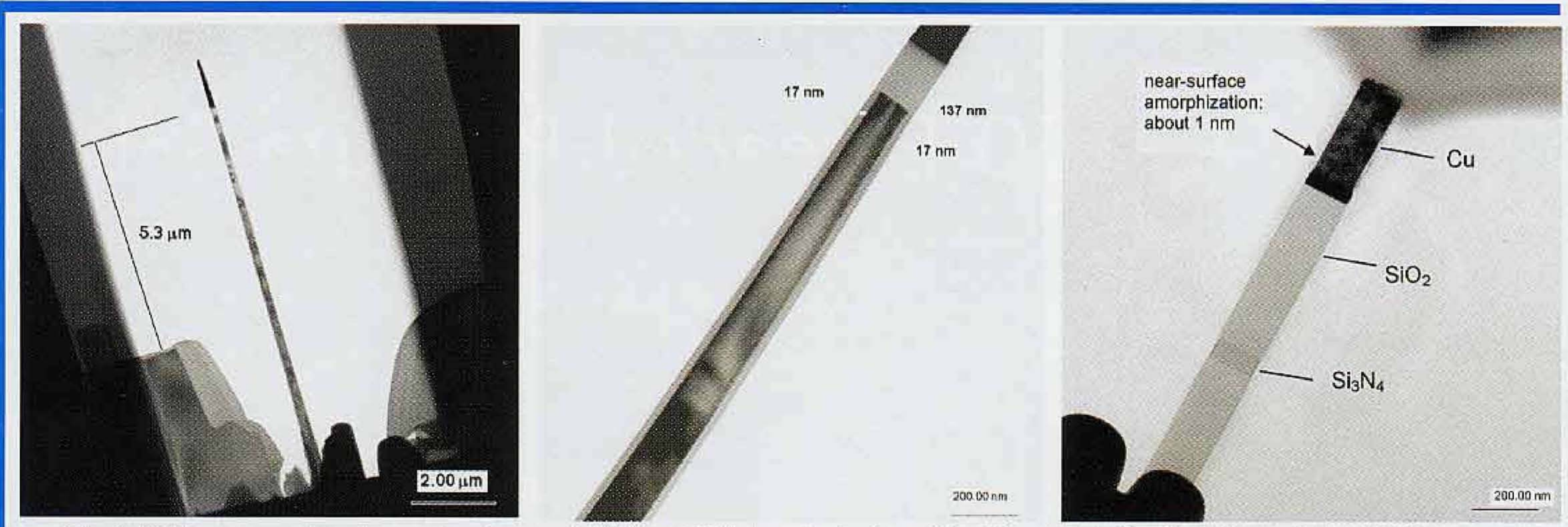

Fig. 4: TEM cross-section of a nearly plan-parallel FIB-cut TEM lamella (on top of the Si material, the Pt cutting edge is visible).

Fig. 5: TEM cross-section of the upper part of the TEM lamella presented in Fig. 4: On both sides, the near-surface amophization is visible.

Fig. 6: TEM cross-section of a FIB-cut TEM lamella consisting of different materials.

final cutting steps such that the standard FIB-cutting pattern cannot be applied any longer. FIB-cuts through the TEM lamella can help to release the stress in the lamella, so that the lamella straightens out almost completely.

The impact of the high energy ions to the near surface region of the TEM lamella is a drawback of the FIB technique. Ions are implanted, structural defects can be induced, and the near-surface region of crystalline materials is amorphized. An alloying effect can be observed, particularly if the solubility for the beam ions in the sample material is low.

Several attempts have been made to minimize the high energy ion beam impact [6]. The authors have analyzed how the near-surface amorphization can be reduced on FIB-cut TEM lamellae by varying $\mathrm{Ga}^{+}$ion energy, ion beam current and sputter angle as well as by subsequent low energy $\mathrm{Ar}+$ ion milling. For all analyses, single crystalline Si was used, which is very sensitive to sputter amorphization. The near-surface amorphization was measured directly at cross-sections of the TEM lamellae.

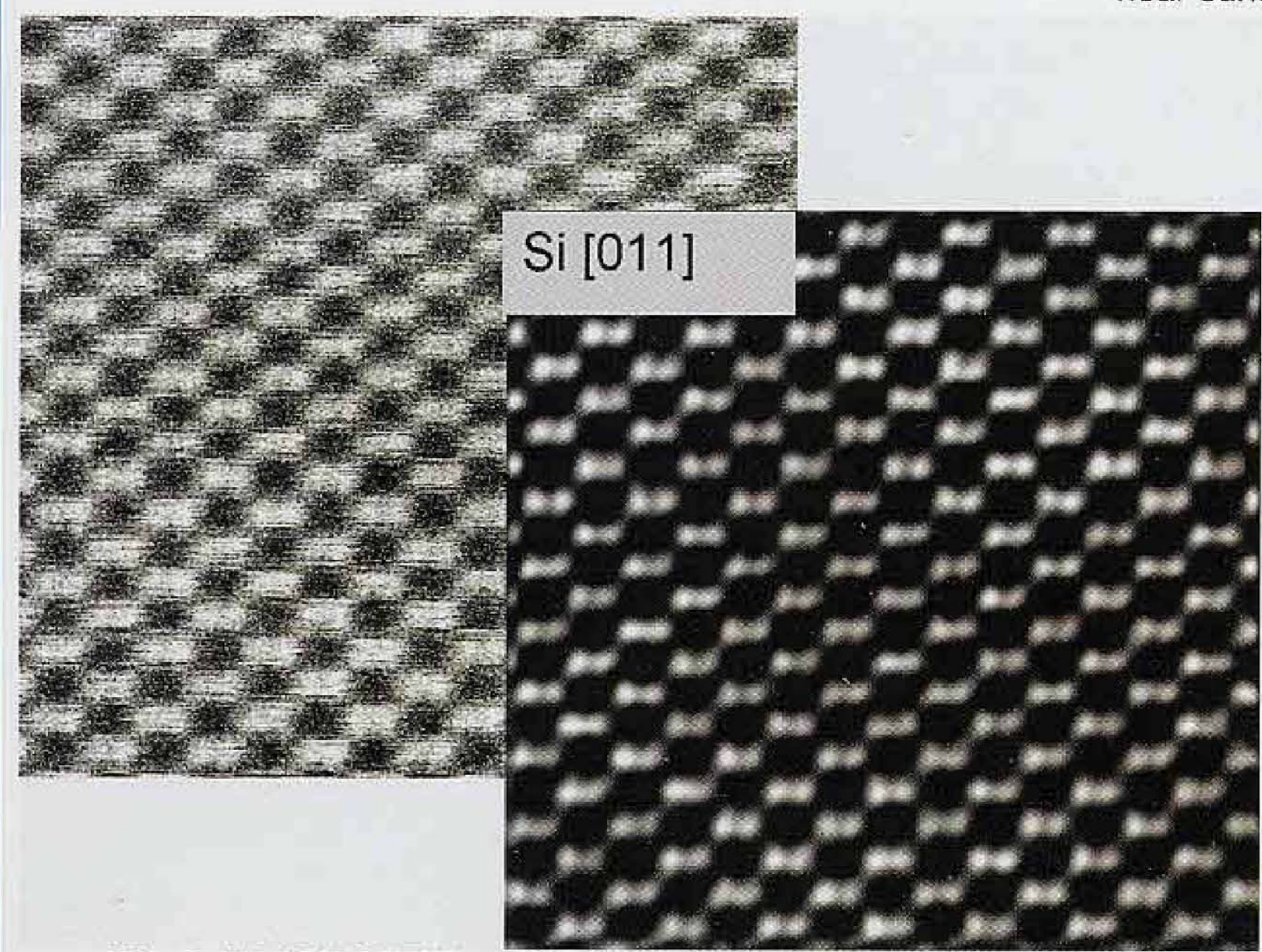

Fig. 7: HAADF-STEM image of Si [011] (unfiltered/low-pass filtered) of a sample prepared with standard FIB parameters and applying $5 \mathrm{keV} \mathrm{Ga}^{+}$ion milling.
Fig. 5 shows the upper part of the TEM lamella presented in Fig. 4. This TEM lamella was prepared applying standard FIB parameters (30 keV Ga+ions). The near-surface amorphization is $17 \mathrm{~nm}$ on each side. It is still under discussion if the amorphization is more dominated by a crystal damage process or by a Si redeposition process. As expected, a relatively high $\mathrm{Ga}$ concentration was detected in these layers by analytical TEM techniques. The thickness of the amorphization layer could not be reduced significantly by decreasing the $\mathrm{Ga}^{+}$ ion energy down to $10 \mathrm{keV}$, reducing the probe current to the lowest possible value of $1 \mathrm{pA}$ or varying the sputter angle. A reduction of the amorphization depth down to $7 \mathrm{~nm}$ was only possible with a $5 \mathrm{keV} \mathrm{Ga+}$ ion beam (the lowest ion energy attainable in the used FIB tool) and tilting the lamella to \pm 7 degree. Subsequent ion milling of the FIB-cut TEM lamella with $3.5 \mathrm{keV} \mathrm{Ar+ions} \mathrm{at} \mathrm{a} \mathrm{tilt} \mathrm{angle} \mathrm{of} 15$ degrees resulted in a near-surface amorphization depth of 5 to $6 \mathrm{~nm}$. This value is close to the near-surface amorphization of $4 \mathrm{~nm}$ that was measured on a classically prepared TEM sample with final $3.5 \mathrm{keV} \mathrm{Ar+}$ ion milling. Using $1.5 \mathrm{keV} \mathrm{Ar}+$ ions and tilting the sample by \pm 20 degrees, the near-surface amorphization is between 2 and $3 \mathrm{~nm}$. Further reduction of the near-surface amorphization is expected using ultralow energy ion milling [5]. For metallic materials, standard FIB-cut TEM lamellae show much thinner near-surface amorphization. In the case of copper, an amorphization depth of only $1 \mathrm{~nm}$ was measured (Fig. 6).

TEM sample preparation using FIB is well suited for many materials and structures. FIB-cut TEM samples are also well suited for most TEM techniques. As an example, in Fig. 7 a high resolution HAADF-STEM image of Si [011] is shown. As recently shown at Electron Microscopy conferences, further development and optimization of the FIBcutting technique for TEM sample preparation is ongoing [7]. It is expected that this unique technique will become a standard technique in materials science soon.

\section{References:}

[1] E.C.G. Kirk, D.A. Williams, H. Ahmed, Inst. Conf. Ser. 100 (7), 501 (1989)

[2] L.A. Giannuzzi, F.A. Stevie, Micron 30, 197 (1999)

[3] J. Orloff, Rev. Sci. Instrum. 64 (5), 1105 (1993)

[4] H.J. Engelmann, B. Volkmann, W. Blum, E. Zschech, Pract. Metallogr. 39 (3), 117 (2002)

[5] H.J. Engelmann, B. Volkmann, E. Zschech, US Patent 6.303.399 B1 (2001)

[6] A. Barna, B. Pecz, M. Menyhard, Micron 30, 267 (1999)

[7] Proc. ICEM15, Durban 2002, Physics and Materials, 247-270. 


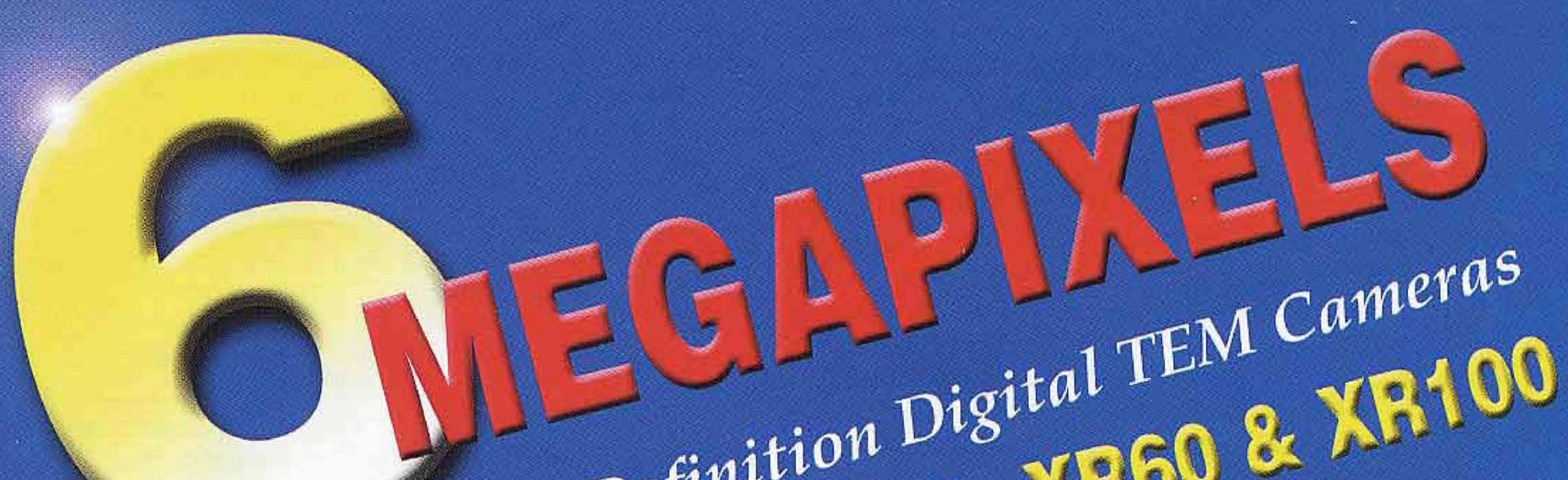

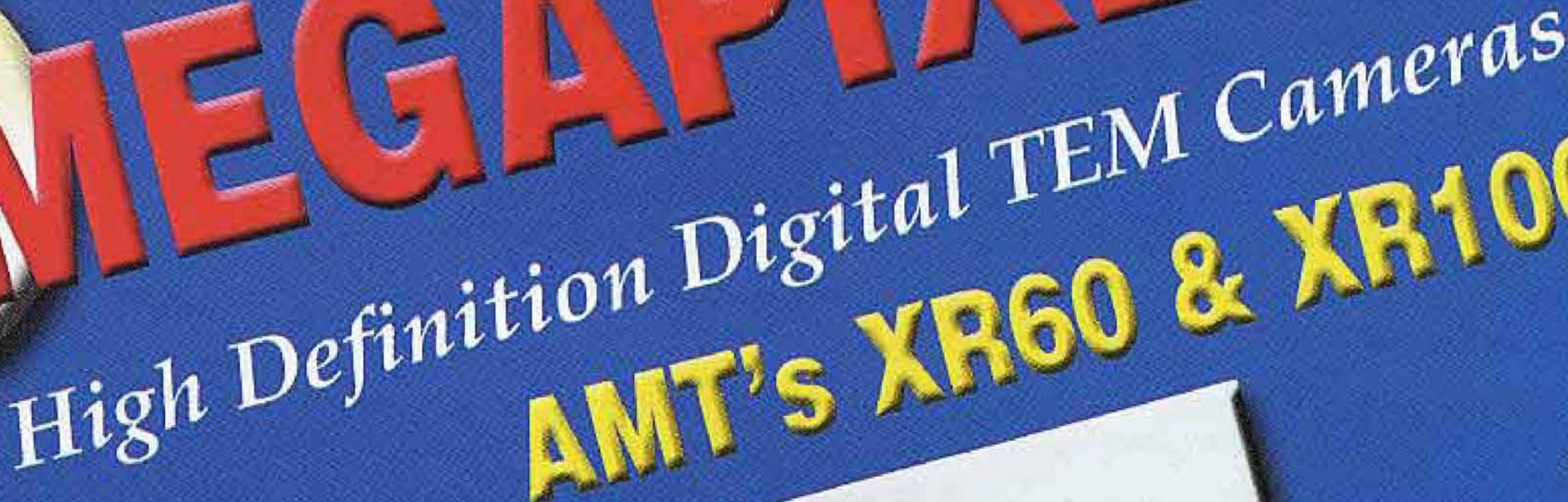
TEM MTEERATION

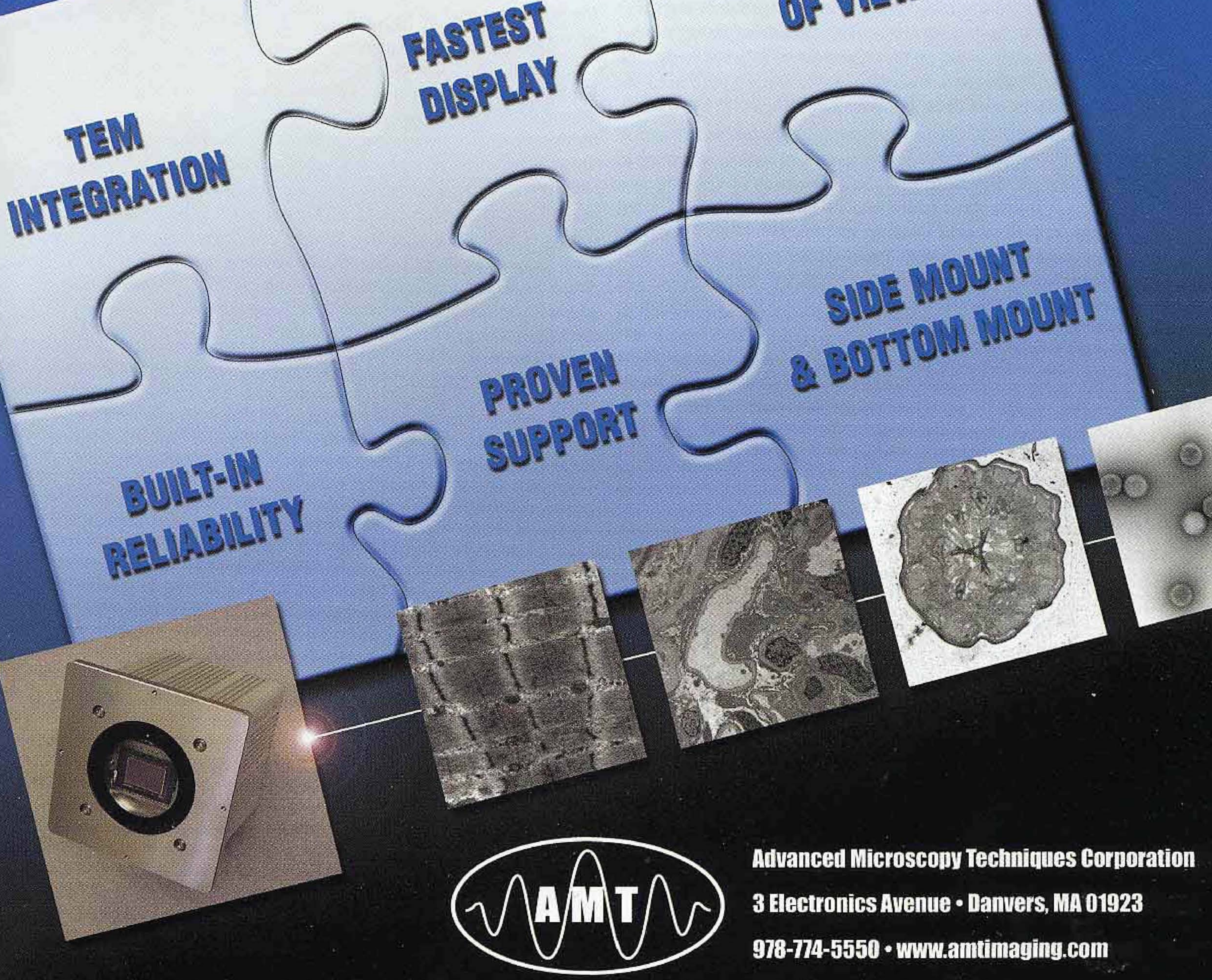

